\title{
Second Toe Plantar Free Flap for Volar Tissue Defects of the Fingers
}

\author{
Yong Jin Cho, Si Young Roh, Jin Soo Kim, Dong Chul Lee, Jae Won Yang \\ Department of Plastic and Reconstructive Surgery, Kwang-Myung Sung-Ae General Hospital, Gwangmyeong, Korea
}

Background The reconstruction of volar surface defects is difficult because of the special histologic nature of the tissue involved. The plantar surface is the most homologous in shape and function and could be considered the most ideal of reconstructive options in select cases of volar surface defects. In this paper, we evaluate a single institutional case series of volar tissue defects managed with second toe plantar free flaps.

Methods A single-institution retrospective review was performed on 12 cases of reconstruction using a second toe plantar free flap. The mean age was 33 years (range, 9 to 54 years) with a male-to-female ratio of 5-to-1. The predominant mechanism was crush injury (8 cases) followed by amputations ( 3 cases) and a single case of burn injury. Half of the indications (6 cases) were for soft-tissue defects with the other half for scar contracture.

Results All of the flaps survived through the follow-up period. Sensory recovery was related to the time interval between injury and reconstruction-with delayed operations portending worse outcomes. There were no postoperative complications in this series.

Conclusions Flexion contracture is the key functional deficit of volar tissue defects. The second toe plantar free flap is the singular flap whose histology most closely matches those of the original volar tissue. In our experience, this flap is the superior reconstructive option within the specific indications dictated by the defect size and location.

Keywords Free tissue flaps / Soft tissue injuries / Finger injuries
Correspondence: Si Young Rho

Department of Plastic and

Reconstructive Surgery,

Kwang-Myung Sung-Ae General

Hospital, 36 Digital-ro, Gwangmyeong

423-711, Korea

Tel: +82-2-2680-7238

Fax: +82-2-2615-7218

E-mail:psczero@gmail.com

Received: 1 Dec 2012 • Revised: 31 Jan 2013 • Accepted: 1 Feb 2013

pISSN: 2234-6163・ elSSN: 2234-6171 • http://dx.doi.org/10.5999/aps.2013.40.3.226・ Arch Plast Surg 2013;40:226-231

This article was presented at the 69th Congress of the Korean Society of Plastic and Reconstructive Surgeons on November 11-13, 2011 in Seoul, Korea.

No potential conflict of interest relevant to this article was reported.

\section{INTRODUCTION}

The volar surface of the upper extremity digits can be divided into two main sections: the pulp tissue of the finger-tip and the proximal soft tissue from over the base of the distal phalanx to the proximal phalanx. Of the two, the latter contains the neurovascular bundle and play the specific role of accommodating interphalangeal joint extension. Such volar soft tissue defects expose critical structures such as the neurovascular bundle, flexor tendons, and phalangeal bones. Left alone, these defects most frequently result in complications such as wound infection and functional disability.

Operative management of these defects includes local, regional, and free tissue flaps. The choice of flap depends on factors such as skin characteristics, defect sizes, donor site morbidity, and facilitation of rehabilitation. Among these determinant factors, the main disadvantage of local/regional flaps is delayed rehabilitation and subsequent joint stiffness [1-3]. In contrast, the main advantage of a free flap is early mobilization. Of the variety of free flaps available in the reconstruction of such volar defects, the 
plantar surface of the second toe offers the ideal tissue with histological equivalency-that of glabrous skin with flexion creases. In this retrospective study, we describe our experience of volar surface reconstruction using the second toe plantar free flap.

\section{METHODS}

\section{Subjects}

A retrospective review of clinical data was performed. The inclusion criterion was all-comers who received plantar free flap at our institution. There were no exclusion criteria. For each of the patients, the following data was collected and tabulated: demographic information (age, sex), injury mechanism and location, indication, tissue defect dimensions, type of donor site coverage, and duration of follow-up. Any available sensory recovery data from follow-up appointments were also collected.

There were 12 cases of second toe plantar free flap. The mean age was 33 years (range, 9 to 54 years) with a male-to-female ratio of 5:1. The most common mechanism of injury was crushing injury ( 8 cases) followed by complete amputation ( 3 cases) and electrical burn ( 1 cases). Half of the indications ( 6 cases) were for flexion contracture; the other half were for the coverage of open defects. In three of the patients, the digital nerve was found to be missing or damaged at the time of surgery (Table 1).

\section{Perioperative details}

In all of the cases, the patient underwent brachial plexus and spinal blocks. A pneumatic tourniquet was applied to the upper arm in the standard fashion. For the delayed indications for the free flap, any preexisting flexion contracture was excised. For immediate reconstruction, non-viable tissue was debrided. The required flap dimensions were measured from the open wound, and the design was made on the plantar surface of the ipsilateral second toe according to those dimensions. A skin incision was made with careful preservation of the subcutaneous vein. The plantar digital nerve on the tibial side was elevated along with the rest of the flap for the three patients who had concomitant neurovascular bundle defects. At the recipient site, the flap was inset. The subcutaneous vein and digital artery were anastomosed. The plantar digital nerve was interposed in the three cases mentioned above. In one case, the donor site was closed with a local flap. In the majority of cases, however, the donor sites were closed with skin grafts taken from within the same operative field-mainly, the medial side of the same toe. Postoperative management consisted of hypervolemic therapy, heparin injections (2,000 U every 4 hours), and prophylactic antibiotics for 1 week. Prostaglandin ( $2 \mathrm{mg}$ daily) was administered for the first five days of this same period.

\section{RESULTS}

The mean area of the repaired defects was $1.4 \times 2.3 \mathrm{~cm}$. The distribution of injuries across the digits were as follows: 5 cases of index finger, 1 case of middle finger, 5 cases of ring finger, and 1 case of little finger. Two cases were in the proximal base of the distal phalanx, 7 in the middle phalanx, and 3 in the proximal phalanx (Table 1). There were no local or general postoperative complications. Neither postoperative venous congestion nor vessel spasm were observed, and consequently, there were no take-back operations for anastomotic revision. The mean follow up period was 242 days (range, 35 to 1,085 days), and all of the flaps survived through this period. No flexion contracture developed in either donor or recipient digits.

For the three cases in which the flap was also used as an inter-

Table 1. Patient profile

\begin{tabular}{|c|c|c|c|c|c|c|c|c|}
\hline Patient & Age/Sex & Injury type & Indication & Location & $\begin{array}{l}\text { Defect size } \\
\quad(\mathrm{cm})\end{array}$ & $\begin{array}{l}\text { Donor site } \\
\text { coverage }\end{array}$ & $\begin{array}{l}\text { Follow-up } \\
\text { (day) }\end{array}$ & $\begin{array}{l}\text { Neurovascular } \\
\text { bundle defect }\end{array}$ \\
\hline 1 & $19 / \mathrm{M}$ & Crush & Soft tissue defect & Rt. RF/MP & $1.5 \times 2.5$ & FTSG & 229 & Present \\
\hline 2 & $36 / \mathrm{M}$ & Crush & Scar contracture & Lt. MF/MP & $2.5 \times 2.5$ & STSG & 95 & Present \\
\hline 3 & $54 / \mathrm{M}$ & Amputation & Scar contracture & Rt. RF/MP & $2 \times 2.5$ & STSG & 117 & Present \\
\hline 4 & $21 / \mathrm{M}$ & Burn & Soft tissue defect & Rt. RF/PP & $2 \times 2$ & STSG & 249 & - \\
\hline 5 & $43 / \mathrm{M}$ & Crush & Scar contracture & Lt. IF/MP & $1.2 \times 2$ & Local flap & 111 & - \\
\hline 6 & $28 / \mathrm{M}$ & Amputation & Soft tissue defect & Lt. IF/MP & $1.5 \times 2.5$ & FTSG & 90 & - \\
\hline 7 & $36 / \mathrm{M}$ & Amputation & Soft tissue defect & Rt. IF/MP & $2 \times 2$ & STSG & 297 & - \\
\hline 8 & $37 / \mathrm{M}$ & Crush & Soft tissue defect & Lt. IF/DP & $1.2 \times 3$ & STSG & 244 & - \\
\hline 9 & $50 / F$ & Crush & Soft tissue defect & Rt. RF/DP & $1 \times 2$ & STSG & 35 & - \\
\hline 10 & 9/F & Crush & Scar contracture & Rt. LF/PP & $2 \times 3$ & STSG & 1,085 & - \\
\hline 11 & 30/M & Crush & Scar contracture & Lt. RF/PP & $3 \times 4$ & STSG & 189 & - \\
\hline 12 & $38 / \mathrm{M}$ & Crush & Scar contracture & Lt. IF/MP & $2 \times 4$ & STSG & 170 & - \\
\hline
\end{tabular}


positional graft, follow-up sensory exams were available (Table 2). At 33 weeks, Patient 1 exhibited a marked sensory recovery in the affected fingertip. Tactile resolutions, both static and dynamic, was indistinguishable between the affected side and the unaffected contralateral side of the same fingertip. Patients 2 and 3 , however, demonstrated no significant sensory recovery, and in these patients, the fingertip sides with neurocutaneous bridge flaps had extremely poor tactile resolution as compared with the contralateral, unaffected sides.

\section{Case 1}

A 19-year-old man presented to the ER with a roller press in-

Table 2. Sensory recovery of 2 nd toe plantar free flap with vascularized nerve graft

\begin{tabular}{|c|c|c|c|c|c|}
\hline Patient & S2PD (mm) & $\mathrm{M} 2 \mathrm{PD}(\mathrm{mm})$ & SWM (size) & Follow-up (wk) & $\begin{array}{c}\text { Interval between injury and } \\
\text { operation (wk) }\end{array}$ \\
\hline Patient 1 & & & & 33 & 0 \\
\hline Affected site (fingertip) & 3 & 3 & 2.83 & & \\
\hline Unaffected site (fingertip) & 3 & 3 & 2.83 & & \\
\hline Affected site (2nd toe plantar flap) & & & 3.61 & & \\
\hline Patient 2 & & & & 14 & 24 \\
\hline Affected site (fingertip) & $>15$ & $>15$ & 6.65 & & \\
\hline Unaffected site (fingertip) & 5 & 5 & 3.61 & & \\
\hline Patient 3 & & & & 20 & 18 \\
\hline Affected site (fingertip) & $>15$ & $>15$ & 6.65 & & \\
\hline Unaffected site (fingertip) & 5 & 5 & 3.61 & & \\
\hline
\end{tabular}

\section{Fig. 1. Patient 1}

$(A, B)$ Volar tissue defect over middle phalanx of right ring finger. (C, D). Flap design. (E) Elevated flap ( $A$, digital artery; $N$, digital nerve; $D / N$, dorsal vein; $V$, subcutaneous vein). (F) Donor site closure. $(G, H)$ Immediate postoperative flap. (I) Reconstructed volar surface at 9-month postoperative visit. (J) Donor site at the same visit.
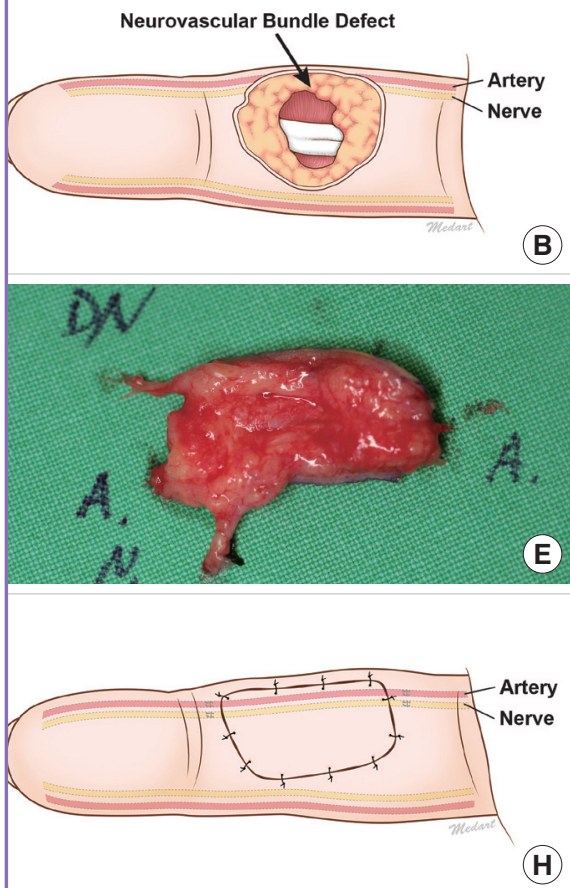
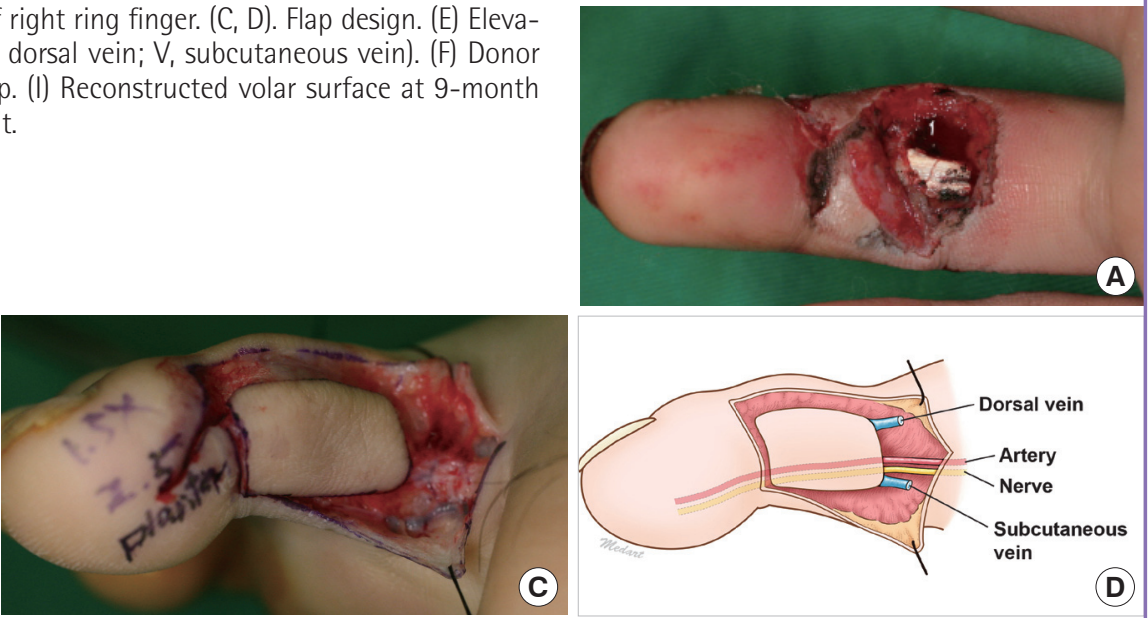

(
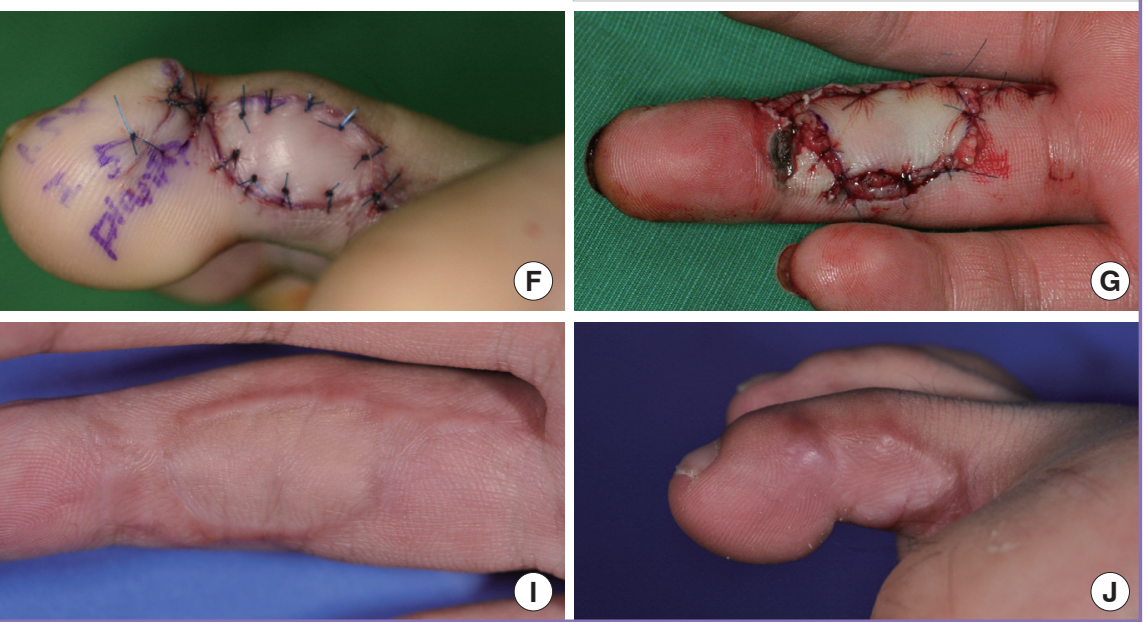
jury to the right ring finger. Examination of the injured finger revealed a $1.5 \times 2.5 \mathrm{~cm}$ volar soft tissue defect over the middle phalanx with accompanying loss of the radial neurovascular bundle (Fig. 1A, B). An emergency single-stage reconstruction was performed. An immediate second toe plantar free flap was performed with the incorporation of the plantar digital nerve as a bridge conduit between the severed ends of the digital nerve (Fig. 1C-E). The donor site was closed with a full thickness graft (Fig. 1F). Postoperatively, the flap showed good circulation (Fig. 1G, H).

At the 9-month follow-up visit, static and dynamic two-point discrimination of the involved fingertip was normal at $3 \mathrm{~mm}$. The Semmes-Weinstein monofilament test showed the injured area to have tactile resolution which equaled that of the noninjured finger on the contralateral side. The range of motion in the involved joint was comparable to the contralateral digit. The flap was similar in shape and color to the surrounding tissue (Fig. 1I, J).

\section{Case 2}

A 21-year-old male suffered a third-degree electrical burn to the right ring finger on the proximal volar surface and underwent post-wound debridement for tissue necrosis. Over the ensuing weeks, the resulting soft-tissue defect developed into a flexion contracture over the proximal interphalangeal joint (Fig. 2A). The contracted scar tissue was removed, and the neurovascular bundle was found to be unaffected.

A plantar free flap transfer was performed as described as above (Fig. 2B, C). The flap artery was anastomosed in an end-to-side fashion. The flap subcutaneous vein was anastomosed to the subcutaneous vein found in the proximal margin of the recipient wound. The digital nerve, which was harvested along with the plantar digital artery, was not anastomosed in this case. The remaining portion of the operation was completed as previously described.

Postoperatively, the patient recovered well without complications. The involved digit showed a full passive range of motion at follow-up visits (Fig. 2D).

\section{DISCUSSION}

A volar surface defect over the interphalageal joints is difficult to address. Ideal reconstruction of such a defect requires skin that is sensate, pliable, and glabrous [4]. A variety of flaps may be

\section{Fig. 2. Patient 4}

(A) Flexion contracture over proximal phalanx and metacarpophalangeal crease. (B) Flap design. (C) Inset flap. (D) At 7-month postoperative clinic visit.
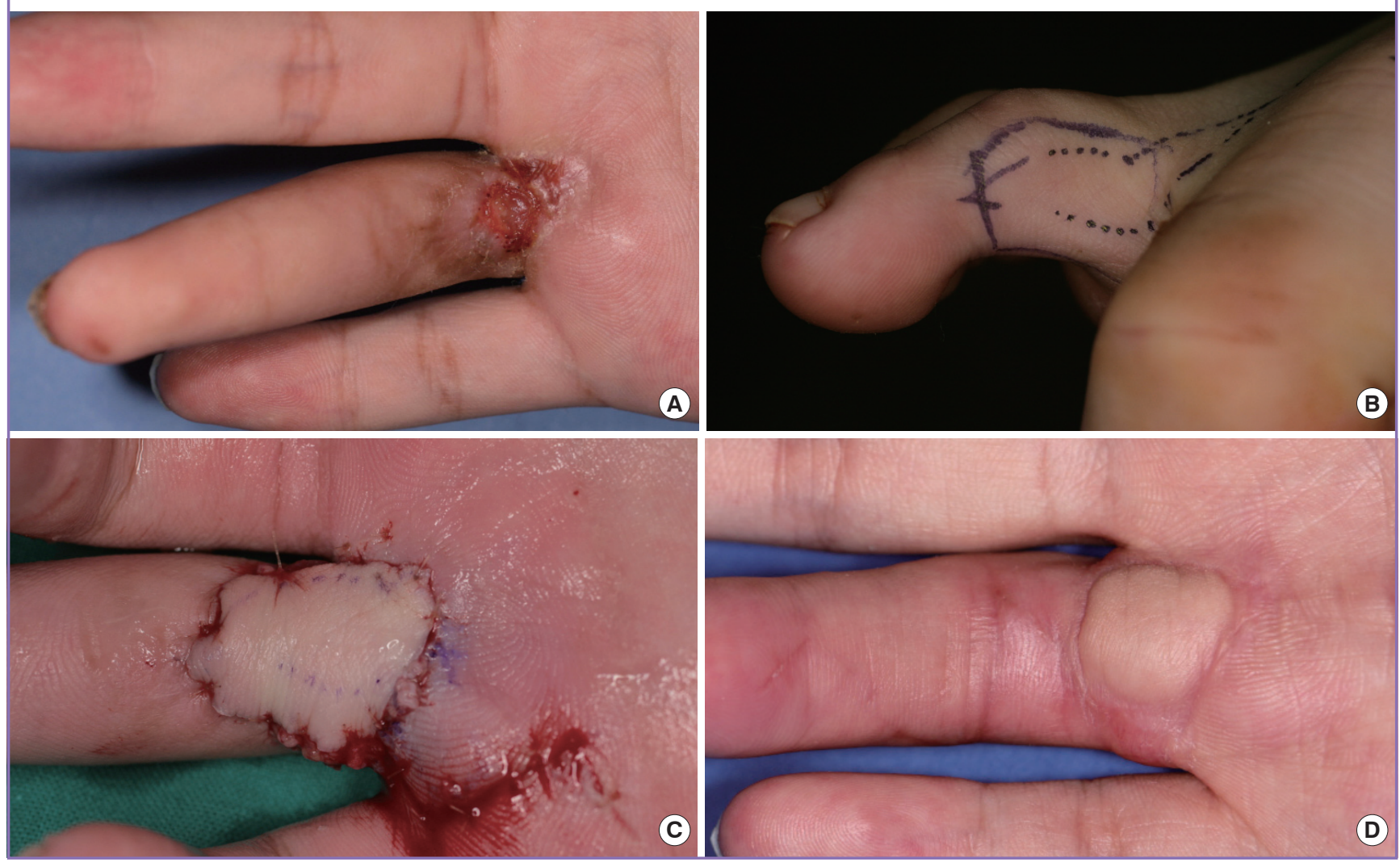
employed in reconstruction of such defects, but in practice, the choice of flap is determined largely by the size and location of the existing defect.

When such a defect is small, local flaps such as thenar and cross-finger flaps are valid options. However, the disadvantage with such an option is the prolonged period of joint immobility with resultant stiffness. Further, this type of reconstructive management requires secondary operations for flap division at a later time, where single stage reconstructions are possible without considerable morbidity $[2,3]$.

For larger defects, the arterialized venous free flap does have the advantage of size. It is functionally and aesthetically inferior to flaps of the palm and the sole and has a non-physiologic blood supply with the potential for partial necrosis $[5,6]$.

Partial toe pulp free flaps have skin properties that are similar to the volar surface and are appropriate for reconstruction of fingertip pulp defects [7]. However, the contour and thickness of toe pulp flaps make for less-than-ideal replacement tissue for those defects over the interphalangeal joints.

A thenar free flap can yield glabrous skin of a significant size, but its principle disadvantages are that it leaves a donor site defect on the palm and that its sensory recovery is poor [8]. These flaps can be designed to incorporate the palmar cutaneous branch of the median nerve [4]. However, this innervated version of the flap still shares the same donor site morbidities inherent in thenar free flaps.

Anterolateral thigh and lateral arm free flaps allow reconstruction of multiple volar defects. They are unnecessarily bulky over the interphalangeal joint and require extensive defatting of the extra subdermal tissue. These flaps are far inferior to flaps containing glabrous skin and are generally reserved for those extensive hand injuries involving multiple digits.

In 1998, Kimata et al. [9] first reported the use of second toe plantar flaps. In this case series, three fingertip defects were reconstructed with the second toe plantar tissue as a neurocutaneous flap. In 2009, Kimura et al. [10] reported the use of this same flap for the reconstruction of interphalangeal volar tissue defects. This latter study described 15 consecutive second toe plantar flaps, which had been performed for two separate indications: 1) fingertip defects and 2) flexion contracture over interphalangeal joints.

Following the idea of "like-with-like", fingertip pulp defects are reconstructed with partial toe pulp flaps at our institution. Likewise, interphalangeal volar surface defects are reconstructed with second toe plantar free flaps. As such, we have conducted this retrospective review for all of the patients who have received second toe plantar free flap for indications related to soft tissues over the interphalangeal volar surface.
The hand injury demographic in our study is similar to the general hand injury population-predominantly male patients of working age. Owing to the retrospective nature of the study design, there is a certain selection bias. This fact is most notable in the mean dimensions of the defect sizes re-surfaced with the flap to be about $2 \mathrm{~cm}$. Though the flap could be as large as $2 \times 4$ $\mathrm{cm}$, the longitudinal dimension is limited to the approximate length of a phalangeal segment. Large volar soft tissue defects, such as those resulting from degloving injuries, cannot be managed with the plantar free flap, and such defects are often reconstructed with thenar free flaps at our institution.

Where the size of the volar tissue defect is limited, however, the plantar toe flap is aesthetically and functionally superior to all other forms of reconstruction. It is pliable enough to allow full passive range of motion. The replacement surface is glabrous and has tactile properties extremely similar to that of the native volar surface.

In three of the cases (patients 1,2, and 3) where the neurovascular bundle was either damaged or missing, we have attempted to incorporate the plantar digital nerve as an interpositional graft into the plantar flap with mixed results. The patient who showed the most marked sensory recovery in the fingertip involved required immediate soft tissue coverage for an open defect. As such, the operation was performed soon after the initial injury with the proximal nerve endings, presumably, still in the reorganizing phase.

In contrast, the two other patients had the plantar flap transfer weeks after the original injury for the primary indication of flexion contracture. At the time of operation, the digital nerve of the injured finger was found to be either damaged or missing, and subsequently, the plantar nerve was interposed as a salvage effort. Unfortunately, the resulting sensory recovery of the involved fingertips was poor in both of these patients. The results of these three interpositional grafts suggest that immediate reconstructions using plantar free flaps are certainly warranted if the initial assessment suggests an injury that may have caused irrecoverable nerve damage.

Regardless of the sensory results, the primary aims of all of the cases were the same-the correction and/or prevention of flexion contracture. This was accomplished in all twelve of the patients with full range of motion restored to each of the involved interphalangeal joints. The secondary aim was to restore the mechanical and aesthetic properties of the glabrous skin to the volar surface of the injured finger. This, too, was accomplished with all of the patients reporting satisfaction with regard to the postoperative "look and feel" of the involved finger.

To the best of our knowledge, our case series represents the first report of plantar free flap purely for the reconstruction of 
interphalangeal volar surface reconstruction. If the size of the volar defect is equal to or less than the length of a phalanx, the second toe plantar free flap is able to restore the aesthetic and functional properties of original volar tissue. In those cases where a digital nerve defect is confirmed or highly suspected, the authors do believe that an immediate plantar flap with an interpositional digital nerve graft could restore sensation to the involved fingertip. With the right indications, plantar free flaps are superior to all other forms of reconstruction in the management of volar soft tissue defects over the interphalangeal joints.

\section{REFERENCES}

1. Cho SB, Choi JK, Lee DC, et al. Reconstruction of the skin defects of the hand using versatile venous free flaps.J Korean Soc Plast Reconstr Surg 1995;22:875-83.

2. Okazaki M, Hasegawa H, Kano M, et al. A different method of fingertip reconstruction with the thenar flap. Plast Reconstr Surg 2005; 115:885-8.

3. Cohen BE, Cronin ED. An innervated cross-finger flap for fingertip reconstruction. Plast Reconstr Surg 1983;72:688-
97.

4. Yang JW, Kim JS, Lee DC, et al. The radial artery superficial palmar branch flap: a modified free thenar flap with constant innervation. J Reconstr Microsurg 2010;26:529-38.

5. Lee GJ, Kim KC, Yu SO, et al. Reconstruction of soft tissue defects of multiple digits with arterialized venous free flap. J Korean Soc Surg Hand 2004;9:12-20.

6. De Lorenzi F, van der Hulst RR, den Dunnen WF, et al. Arterialized venous free flaps for soft-tissue reconstruction of digits: a 40-case series. J Reconstr Microsurg 2002;18:569-74.

7. Lee DC, Kim JS, Ki SH, et al. Partial second toe pulp free flap for fingertip reconstruction. Plast Reconstr Surg 2008; 121:899-907.

8. Kamei K, Ide Y, Kimura T. A new free thenar flap. Plast Reconstr Surg 1993;92:1380-4.

9. Kimata Y, Mukouda M, Mizuo H, et al. Second toe plantar flap for partial finger reconstruction. Plast Reconstr Surg 1998;101:101-6.

10. Kimura N. Versatility of a second toe plantar flap. J Reconstr Microsurg 2009;25:47-53. 